\title{
Dificuldades na implementação da Sistematização da Assistência de Enfermagem na Unidade de Terapia Intensiva Adulto
}

\author{
Difficulties in the implementation of Nursing Assistance Systematization in the Adult \\ Intensive Care
}

\begin{abstract}
Dificultades em la implementación de la Sistematización de la Assistência de Enfermeira em la Unidad de Terapia Intensiva Adulto
\end{abstract}

Francisco Mateus Lima da Silva ${ }^{1 *}$, José João Morais de Carvalho ${ }^{1}$, Luciene Carvalho Piedade Almeida ${ }^{1}$.

\section{RESUMO}

Objetivo: Identificar as dificuldades na implementação da Sistematização da Assistência de Enfermagem na Unidade de Terapia Intensiva adulto no município de Porto Velho - RO. Métodos: Trata-se de uma pesquisa bibliográfica, de campo, descritiva, exploratória, de caráter qualitativa. A amostra da pesquisa foi constituída por 21 profissionais de enfermagem (5 enfermeiros e 16 técnicos de enfermagem), atuantes na Unidade de Terapia Intensiva adulto do Hospital e Pronto Socorro João Paulo II, utilizando-se um questionário com perguntas fechadas para caracterização profissional e seis (06) perguntas abertas focadas no tema central. A pesquisa foi aprovada pelo Comitê de Ética em Pesquisa com o Parecer de no: 3.180.102. Resultados: Os resultados mostraram uma sobrecarga de trabalho por parte dos enfermeiros e conhecimento superficial da equipe de enfermagem a respeito da SAE. Conclusão: É preciso que as condições no ambiente de trabalho favoreçam a qualidade no serviço de enfermagem prestado, como o número de profissionais enfermeiros adequado frente às demandas de serviços assistenciais e burocráticos, além do conhecimento profundo do tema com discussões e capacitações em serviço através da educação permanente com os profissionais para que os enfermeiros e técnicos de enfermagem reconheçam seus papéis na sistematização.

Palavras-chave: Sistematização, Enfermagem, Educação.

\begin{abstract}
Objective: To identify the difficulties in the implementation of Nursing Care Systematization in the Adult Intensive Care Unit of the city of Porto Velho - RO. Methods: This is a qualitative, bibliographic, descriptive, exploratory and field study. The research sample consisted of 21 nursing professionals (5 nurses and 16 nursing technicians), who worked in the Adult Intensive Care Unit of the Hospital and Emergency Room João Paulo II, using a questionnaire with closed questions for professional characterization and six (06) directed questions focused on the central theme. The research was approved by the Research Ethics Committee with opinion $n=3,180,102$. Results: The results showed an overload of nurses' work and superficial knowledge of the nursing team regarding SAE. Conclusion: It is necessary that conditions in the work environment favor quality in the nursing service provided, such as the number of nurses adequate to the demands of care and bureaucratic services, as well as the in-depth knowledge of the topic with discussions and in-service training through permanent education with professionals so that nurses and nursing technicians recognize their roles in systematization.
\end{abstract}

Key words: Systematization, Nursing, Education.

${ }^{1}$ União das Escolas Superiores de Rondônia (UNIRON), Porto Velho-RO. *E-mail: franciscomateus95@hotmail.com 


\section{RESUMEN}

Objetivo: Identificar las dificultades en la implementación de la Sistematización de la Asistencia de Enfermería en la Unidad de Terapia Intensiva adulto en el municipio de Porto Velho - RO. Métodos: Se trata de una investigación bibliográfica, de campo, descriptiva, exploratoria, de carácter cualitativo. La muestra de la investigación fue constituida por 21 profesionales de enfermería (5 enfermeros y 16 técnicos de enfermería), actuantes en la Unidad de Terapia Intensiva adulto del Hospital y Pronto Socorro João Paulo II, utilizando un cuestionario con preguntas cerradas para caracterización profesional y seis (06) preguntas abiertas enfocadas en el tema central. La encuesta fue aprobada por el Comité de Ética en Investigación con el Dictamen de no: 3.180.102. Resultados: Los resultados mostraron una sobrecarga de trabajo por parte de los enfermeros y conocimiento superficial del equipo de enfermería respecto a la SAE. Conclusión: Es necesario que las condiciones en el ambiente de trabajo favorezcan la calidad en el servicio de enfermería prestado, como el número de profesionales enfermeros adecuado frente a las demandas de servicios asistenciales y burocráticos, además del conocimiento profundo del tema con discusiones y capacitaciones en servicio a través educación permanente con los profesionales para que los enfermeros y técnicos de enfermería reconozcan sus papeles en la sistematización.

Palabras clave: Sistematización, Enfermería, Educación.

\section{INTRODUÇÃO}

O Conselho Federal de Enfermagem (COFEN) (2009), por meio da Resolução 358/2009, dispõe sobre Sistematização de Assistência de Enfermagem (SAE) e a implementação do Processo de Enfermagem (PE), que deve ser realizado de modo deliberado e sistemático em todos os ambientes públicos e privados em que ocorrer o cuidado profissional de enfermagem.

A SAE contribui não só para a saúde dos pacientes, mas torna o ambiente de trabalho mais dinâmico para os profissionais. Quando implementada corretamente, produz um aumento da autoestima entre a equipe de enfermagem, além de um melhor gerenciamento do processo saúde-doença, pois organiza, direciona, confere autonomia aos profissionais de enfermagem, respaldo jurídico e, aos pacientes, segurança (SANTOS WN, 2014).

O trabalho da enfermagem na Unidade de Terapia Intensiva (UTI) é complexo e, como tal, comporta inúmeras necessidades para o desenvolvimento do cuidado, tendo o enfermeiro um importante papel (MASSAROLI R et al., 2015).

A SAE vem como uma importante metodologia na UTI, pois, através dela, torna possível a operacionalização do PE, onde o enfermeiro planeja e executa ações juntamente com sua equipe (técnicos ou auxiliares), de acordo com as necessidades de cada paciente (SANTOS JS et al., 2014).

Ao planejar a assistência, o enfermeiro deve realizar a supervisão da equipe, principalmente, em relação aos cuidados, visando a eficácia da assistência minimizando, assim, erros e imperícias (BARBOSA TP et al., 2014).

O PE está inserido na SAE e é um processo de cuidar desenvolvido pelos profissionais da equipe de enfermagem, cabendo, a cada um, suas devidas atribuições, sendo necessário que o profissional tenha conhecimentos técnicos e científicos o suficiente, reforçado com uma prática baseada em evidências (GARCIA TR, 2016).

Embora a responsabilidade da SAE e PE estejam claros em sua legislação, ainda percebemos que há dúvidas sobre sua realização entre os membros da Equipe de Enfermagem, e, também, uma inversão de responsabilidades, onde, muitas vezes, a tomada de decisão se dá pelo Técnico ou Auxiliar de enfermagem, reforçando, mais uma vez, a importância de se investir nessas capacitações aos Enfermeiros através de educação continuada (SIMÕES CRF, et al., 2017). 
O interesse pela pesquisa surgiu a partir da experiência vivenciada, pelos pesquisadores, durante a prática hospitalar do curso de enfermagem na Unidade de Terapia Intensiva Adulto do Hospital de estudo, oportunidade na qual, empiricamente, observou as dificuldades da equipe de enfermagem em implementar a SAE. Consideramos importante a compreensão, pelos acadêmicos de enfermagem, acerca dos fatores que dificultam a implementação da SAE e operacionalização do PE visto que, futuramente, serão esses os profissionais que utilizarão desta ferramenta para prestar assistência aos pacientes.

Aos profissionais que já trabalham na área, a pesquisa torna-se importante para fomentar a autorreflexão por parte de cada membro da equipe de enfermagem frente às suas condutas na execução do trabalho assistencial, uma reflexão que consideramos necessária, pois pode levar a uma melhor assistência aos pacientes que se encontram internados nas unidades hospitalares.

Tomamos, então, como objetivo, identificar as dificuldades na implementação da Sistematização da Assistência de Enfermagem na Unidade de Terapia Intensiva Adulto no município de Porto Velho-RO.

\section{MÉTODOS}

Trata-se de uma pesquisa bibliográfica e de campo, descritiva e exploratória, de caráter qualitativa, cuja população de estudo foi composta por enfermeiros e técnicos em enfermagem com o objetivo de identificar as dificuldades para a implementação da sistematização da assistência de enfermagem ao paciente de unidade de terapia intensiva.

O estudo foi executado em duas etapas, sendo a primeira etapa: realização do levantamento de informações por meio de revisão bibliográfica para contextualização referente à temática central SAE; e a segunda etapa: realização da pesquisa de campo com aplicação de um instrumento de coleta de dados (questionário) com perguntas fechadas, apenas, para a caracterização profissional e com 6 perguntas abertas sobre o tema central direcionadas aos participantes da pesquisa.

Para análise dos dados, utilizamos a metodologia proposta por Minayo, através da pré-análise, onde ocorre a organização do material para que se torne útil à pesquisa, leitura flutuante para o conhecimento do conteúdo e familiaridade, e também deverá estabelecer objetivos, ou seja, a preparação do material. $\mathrm{Na}$ segunda etapa, as unidades de codificação são definidas, de acordo com o processo de escolha das categorias, classificação e categorização.

A amostra da pesquisa foi constituída por 21 profissionais de enfermagem (sendo 5 enfermeiros e 16 técnicos de enfermagem), atuantes na Unidade de Terapia Intensiva (UTI) adulto do Hospital e Pronto Socorro João Paulo II. As falas dos profissionais enfermeiros foram identificadas pela letra " $E$ " e as falas dos técnicos de enfermagem pela letra "T". As falas atribuídas aos profissionais enfermeiros foram categorizadas de E1 a E5, pois participaram da pesquisa 5 enfermeiros. Aos técnicos de enfermagem, categorizaram-se as falas entre T1 a T16, pois participaram da pesquisa 16 técnicos de enfermagem.

A pesquisa foi aceita pelo Comitê de Ética e Pesquisa (CEP) com o número de CAAE: 05695018.7.0000.8028 e Parecer de no: 3.180.102. Foram respeitadas as disposições contidas na Resolução de № 466/2012 do Conselho Nacional de Saúde (CNS) que trata das pesquisas com seres humanos.

\section{RESULTADOS E DISCUSSÕES}

\section{Caracterização dos participantes da pesquisa}

Entre os profissionais enfermeiros, 4 foram do sexo feminino e 1 do sexo masculino, sendo a idade média de 33 anos (entre 30 a 37), com o tempo de formação entre 4 a 13 anos e o tempo de lotação na UTI entre 4 meses a 12 anos.

Entre os profissionais técnicos de enfermagem, 11 foram do sexo feminino e 5 do sexo masculino, sendo a idade média de 35 anos (entre 23 a 50), com o tempo de formação entre 2 a 29 anos e o tempo de lotação na UTI entre 4 meses a 15 anos. 
A partir da análise das falas, tendo como base relatos semelhantes e convergentes, foram elencados dois núcleos temáticos: A sobrecarga de trabalho como fator que dificulta a implementação da SAE e Conhecimento superficial da equipe de enfermagem a respeito da SAE.

\section{A sobrecarga de trabalho como fator que dificulta a implementação da SAE}

"Atualmente, conta com um enfermeiro cuja demanda assistencial e administrativo são prioritárias, apenas um computador para o uso de todos os servidores (técnicos, apoio, secretária e enfermeiro)." (E1)

\section{"[..] sobrecarga de trabalho." (E3) UTI/JP II [..]." (E4) \\ "Poucos enfermeiros para uma alta demanda de pacientes." (T16)}

"Sobrecarga do enfermeiro demanda grande atenção; rotatividade/dinâmica da

Devido ao grande número de atividades administrativas, o enfermeiro acaba deixando, para segundo plano, a assistência integral ao paciente, não porque ele quer, mas pelas condições que seu trabalho exige. Os enfermeiros, muitas das vezes, precisam realizar atividades burocráticas de outros profissionais, ou, quando não, de seu próprio serviço, desviando-se de suas ações assistenciais (CARVALHO ML, et al., 2013). No entanto, o mesmo autor não descarta a importância das atividades administrativas para o serviço, pois estão interligadas com as atividades assistenciais, visto que o enfermeiro possui um papel de chefe, líder e administrador dos serviços de enfermagem.

Neste sentido, os enfermeiros possuem conhecimento cientifico e até sabem aplicar o PE, no entanto, por terem que dedicar tanto tempo às atividades burocráticas, ocasionam uma fragmentação do cuidado, além de terem pouco tempo para realizar a sistematização em sua integralidade (NECO KCS, et al., 2015). Visando uma melhor qualidade da assistência aos pacientes, vemos que as instituições de saúde precisam buscar melhores condições de trabalho aos profissionais com vistas à implementação de métodos de organização da assistência de enfermagem, não pensando em si, mas com o objetivo de prestar um cuidado de mais qualidade aos pacientes, favorecendo o seu retorno à vida social e família (CASTRO RR, et al., 2016).

Diante do exposto, nota-se que a sobrecarga de trabalho assistencial e administrativa é apontada como um fator que dificulta a implementação do PE de forma eficaz. Para Gomes LA e Brito DS (2012), a sobrecarga de trabalho também reflete na forma de agir dos profissionais, pois passam a agir de forma repetitiva, mecanizados, restritos à burocracia, deixando de atentarem-se às características individuais de cada paciente, interferindo diretamente na qualidade da assistência.

Não sendo, apenas, a sobrecarga de trabalho que se divide entre a assistência, gerência da equipe e atividades administrativas, vemos que o participante (T16) nos traz outro dado importante, onde não tem sido feito um dimensionamento correto dos profissionais enfermeiros na terapia intensiva, conforme a Resolução do COFEN de № 543/2017 que trata do dimensionamento do Quadro de Profissionais de enfermagem nos serviços e locais em que são realizadas atividades de enfermagem, contribuindo para que a sobrecarga aumente e implique na implementação da SAE no setor e aplicação do PE de forma integral a cada paciente, transformando-se em um dos principais obstáculos para a implementação da SAE (COFEN, 2017).

Corroborando com a afirmação acima, Piccinini VM, et al. (2018) afirmam que um dos fatores que dificultam a implementação do PE pelos enfermeiros é o número reduzido de profissionais e que consequentemente resultam na sobrecarga de trabalho. O mesmo autor cita a falta de conhecimento da equipe como um dos obstáculos a serem trabalhados com a equipe de enfermagem.

\section{Conhecimento superficial da equipe de enfermagem a respeito da SAE e PE}

Outro dado e não menos importante que a sobrecarga de trabalho, foi a falta de conhecimento profundo do instrumento de sistematização, corroborando com o que diz Fernandes VS, et al. (2017) onde além da sobrecarga de trabalho e burocracias, a falta de conhecimento é um dos fatores que implicam para a implementação do PE. 
Apesar dos profissionais de enfermagem, em sua maioria, conhecerem e compreenderem a importância da sistematização no referido setor, constatou-se que o conhecimento deles sobre o PE é superficial. Apenas um profissional (T13) referiu as 5 etapas do processo de enfermagem:

"[..] de suma importância para desenvolver uma assistência adequada, se seguida corretamente as cincos etapas." (T13)

Marinelli NP (2016) nos traz que uma das principais dificuldades para se implementar o PE em sua integralidade, seguindo as 5 etapas, é justamente a falta de conhecimento por parte dos profissionais, especificamente os enfermeiros, visto que os diagnósticos de enfermagem e planejamento dos cuidados são de sua inteira responsabilidade, de acordo com a Resolução 358/2009 que dispõe sobre a SAE em ambientes públicos e privados em que se tem cuidados de enfermagem.

Durante a análise dos dados, encontramos trechos que remetem ao exposto pelo autor acima, pois todos os enfermeiros que responderam o questionário possuíam conhecimento sobre o PE e sua importância no serviço por terem sido instruídos durante sua graduação, no entanto, nenhum enfermeiro referiu as 5 etapas do processo de enfermagem, nos revelando o conhecimento superficial desta ferramenta tão essencial ao gerenciamento do cuidado.

Um profissional respondeu que não achava o SAE importante (T7) e outro preferiu deixar em branco, talvez porque não possuía domínio sobre a sistematização de enfermagem e sua importância.

$$
\begin{aligned}
& \text { "Não, pois não sei a importância." (T7) } \\
& \text { "[...] pouco conhecimento da equipe." (T11)" }
\end{aligned}
$$

As falas acima reforçam a necessidade de melhorar os processos conceituais acerca da SAE e PE através de educação permanente, pois é preciso que os enfermeiros e sua equipe compreendam a SAE em sua totalidade para que a assistência aos pacientes, por meio do processo de enfermagem, não seja prestada de forma fragmentada, visto que o enfermeiro e o técnico de enfermagem estão inseridos dentro da sistematização e execução do processo de enfermagem, cabendo o diagnóstico das necessidades e planejamento dos cuidados ao enfermeiro e execução também aos técnicos.

Ribeiro GS e Padoveze MC (2018) nos trazem que o desconhecimento da SAE, por parte dos profissionais, muito se deve à formação deficitária, tanto na graduação, técnica ou auxiliar, tornando-se necessária a revisão das grades curriculares, pois ainda há uma lacuna entre o ensino e prática dos profissionais de enfermagem.

Abaixo, percebemos que a necessidade de educação permanente em SAE é apontada, pelos próprios profissionais, como algo essencial ao serviço:

"Através da capacitação, os colaboradores vão entender a importância da SAE para a equipe e o paciente." (E4)

"[...] o treinamento nos dá mais segurança e capacitação para podermos desempenhar melhor a nossa função." (T3)

O apoio das instituições de saúde é muito importante à assistência de enfermagem, pois é ela quem viabiliza os recursos necessários à sua implementação e manutenção no serviço. Barbosa $A A$, et al. (2018) nos diz que as discussões, acerca da SAE, devem ser mais constantes nos ambientes hospitalares para um melhor esclarecimento do papel da equipe de enfermagem na sistematização da assistência.

Conforme o relato do profissional (T3), há uma insegurança no desempenho de funções dentro da sistematização, justamente pela falta de conhecimento de seus próprios deveres. Nota-se que ainda há uma interpretação equivocada sobre o processo de enfermagem. A Resolução 358 de 2009 nos traz a atribuição de cada profissional na realização da sistematização, onde o profissional técnico e auxiliar em enfermagem possuem extrema importância na execução dos cuidados de enfermagem prescritos pelo enfermeiro.

Os profissionais necessitam de uma qualificação constante para prestarem uma assistência de qualidade aos pacientes, principalmente na UTI por ser considerado um setor de grande complexidade. 
Martins FR, et al. (2019) afirma justamente isso quando enfatiza que, mesmo se os hospitais tivessem os equipamentos e um quantitativo profissional de enfermagem considerado ideal, não seria o suficiente. Tornando-se importante as qualificações com os profissionais com objetivo de melhorar a assistência aos pacientes e torná-la de forma mais segura.

Já Ferreira AF, et al. (2017) fala do poder que os profissionais possuem, em seu ambiente de trabalho na busca de soluções para os problemas enfrentados, sendo a educação permanente um importante meio para a conscientização e participação destes profissionais no processo de transformação da realidade, ou seja, a capacitação traria conhecimento e reflexões acerca do cuidado ao paciente, tendo maior probabilidade de mudanças no serviço, sendo os profissionais os protagonistas das transformações.

. Para Lemos CS, et al. (2017) o enfermeiro não consegue realizar a SAE aplicando todas as etapas do processo de enfermagem, de forma organizada, por uma série de motivos, como a sobrecarga de trabalho, 0 desconhecimento do instrumento e sua importância para os próprios profissionais, ambiente de trabalho e, principalmente, aos pacientes, ou seja, os resultados encontrados no trabalho vão ao encontro da literatura.

\section{CONCLUSÃO}

É preciso que as condições no ambiente de trabalho favoreçam a qualidade no serviço de enfermagem prestado, como o número de profissionais enfermeiros adequado frente às demandas de serviços assistenciais e burocráticos para que os enfermeiros não dediquem tanto tempo às atividades administrativas e as atividades assistenciais acabem ficando para segundo plano, quando se é preciso avaliar o paciente como um todo, não sendo possível realizar isto quando o tempo está reduzido, o que interfere diretamente na qualidade da assistência da enfermagem. Também é preciso um conhecimento profundo do tema com discussões e capacitações em serviço através da educação permanente com os profissionais para que os enfermeiros e técnicos de enfermagem reconheçam que seus papéis na sistematização são importantes para a garantia de um bom atendimento ao paciente e gerenciamento do cuidado.

\section{REFERÊNCIAS}

1. BARBOSA AA, et.al. Implantação da Sistematização da Assistência de Enfermagem - SAE, no Serviço de Enfermagem: Revisão Integrativa. Revista Científica Multidisciplinar Núcleo do Conhecimento. Ano 03, Ed. 03, Vol. 01, pp. 102-109, março de 2018.

2. BARBOSA TP, et al. Práticas assistenciais para segurança do paciente em unidade de terapia intensiva. Acta Paul Enferm. 27(3):243-8, 2014.

3. CASTRO RR, et al. Compreensões e desafios acerca da sistematização da assistência de enfermagem. Rev enferm UERJ, Rio de Janeiro, 24(5): e10461. 2016.

4. CARVALHO M L, et al. Assistência de enfermagem na UTI a pacientes submetidos à cirurgia cardíaca. R. Interd. v.6, n.4, p.60-67, out.nov.dez. 2013.

5. FERNANDES VS, et al. Dificuldades na implementação da sistematização da assistência de enfermagem em unidade de terapia intensiva.

6. FERREIRA AF, et al. Educação permanente como instrumento para melhorar a qualidade do registro de enfermagem: Revisão integrativa. Revista Pró-univerSUS. 2017 jul./dez.; 08 (2): 28-34. Acesso em: 08 de maio de 2019.

7. GARCIA TR. Sistematização da assistência de enfermagem: aspecto substantivo da prática profissional. Esc Anna Nery, 20(1):5-10, 2016.

8. GOMES LA. BRITO DS. Desafios na implantação da sistematização da assistência de enfermagem: uma revisão de literatura. Rev. Interdisciplinar UNINOVAFAPI. Teresina, v.5, n.3, p.64-70, jul./set. 2012.

9. LEMOS CS, et al. Construção e validação de um protocolo assistencial de enfermagem em anestesia. Rev. LatinoAm. Enfermagem. 25:e2952, 2017.

10. MARTINS FR, et al. Necessidades de qualificação do processo de trabalho da enfermagem em uti pediátrica. Id online Rev. Mult. Psic. V.13, N. 43, p. 322-328, 2019.

11. MASSAROLI R, et al. Trabalho de enfermagem em unidade de terapia intensiva e sua interface com a sistematização da assistência. Esc Anna Nery 19(2) Abr-Jun 2015. 
12. MARINELLI NP, et al. Sistematização da assistência de enfermagem: desafios para implantação. Revista Enfermagem Contemporânea.4(2):254-263, Jul./Dez.; 2015.

13. NECO KCS, et al. Sistematização da assistência de enfermagem em instituições de saúde no brasil: revisão integrativa. Rev enferm UFPE on line., Recife, 9(1):193-200, jan., 2015.

14. PICCININI VM, et al. Implantação da sistematização da assistência de enfermagem como meio de qualificação da assistência ao idoso. RBCEH, Passo Fundo, v. 14, n. 3, p. 307-317, set./dez. 2017.

15. Resolução Cofen - 358/2009. Publicado no Portal do COFEN - Conselho Federal de Enfermagem.

16. Resolução Cofen - 543/2017. Publicado no Portal do COFEN - Conselho Federal de Enfermagem.

17. RIBEIRO GS. PADOVEZE MC. Sistematização da assistência de enfermagem em unidade básica de saúde: percepção da equipe de enfermagem. Rev Esc Enferm USP, 52:e03375, 2018.

18. SANTOS JS, et al. Sistematização da Assistência de Enfermagem na Unidade de Terapia Intensiva: Revisão Bilbliográfica. Ciências Biológicas e da Saúde. Aracaju. v. 2, n.2 [p. 45-58] 2014.

19. SANTOS WN. Sistematização da assistência de enfermagem: o contexto histórico, o processo e obstáculos da implantação. J Manag Prim Health Care, 5(2):153-158, 2014.

20. SIMÕES CRF, et al. A equipe de enfermagem frente a sistematização da assistência de enfermagem. Revista científica revela Edição 21 - Dezembro de 2017. 